\title{
Early labour assessment improved several intrapartum outcomes
}

\author{
McNiven PS, Williams JI, Hodnett E, et al. An early labor assessment program: a randomized, controlled trial. Birth 1998 \\ Mar;25:5-10.
}

\section{Question}

Can early labour assessment improve intrapartum outcomes?

\section{Design}

Randomised controlled trial.

\section{Setting}

A large teaching hospital in southwestern Ontario, Canada.

\section{Patients}

209 women (mean age 25 y) who were $\geqslant 37$ weeks gestation, had no previous live births, were identified as low risk according to the Ontario antenatal record, and who had spontaneous labour.

\section{Intervention}

105 women were allocated to early assessment of labour status (active or latent). Active labour was defined as the presence of regular, painful contractions and cervical dilation $>3 \mathrm{~cm}$. Those not in active labour were provided with encouragement and advice and told to return home or walk outside until labour became more active. 104 women were allocated to direct hospital admission. All women received usual intrapartum care.

\section{Main outcome measures}

Rates of caesarean section birth; oxytocin, epidural, or narcotic use, forceps delivery; vacuum extraction; amniotomy; labour duration and experience; and newborn outcomes.

\section{Main results}

Analysis was by intention to treat. Women who received early labour assessment used less oxytocin, epidurals, or narcotics (table); had shorter durations between admission and delivery (8.3 $v 13.5 \mathrm{~h}, \mathrm{p}=0.001)$ and between full dilation and delivery (77 $v 95 \mathrm{~min}, \mathrm{p}=0.045$ ); and more positive experiences (higher Labor Agentry Scale scores, 158 v 142, $\mathrm{p}=0.001)$ compared with women who were admitted directly. No differences existed for caesarean section births, forceps delivery, vacuum extraction, or amniotomy (table); or newborn outcomes.

\section{Conclusion}

Early labour assessment did not decrease caesarean section births but decreased oxytocin, epidural, or narcotic use, and labour duration; and improved the labour experience.

Early labour assessment (ELA) v direct admission (DA)*

\begin{tabular}{lllll}
\hline Outcomes & ELA & DA & RRR (95\% CI) & NNT (CI) \\
\hline Caesarean section & $8 \%$ & $11 \%$ & $28 \%(-67$ to 69$)$ & NS † \\
Oxytocin use & $23 \%$ & $40 \%$ & $43 \%(14$ to 63$)$ & $6(3$ to 20$)$ \\
Epidural or narcotic use & $80 \%$ & $92 \%$ & $13 \%(4$ to 23$)$ & $8(5$ to 33$)$ \\
Forceps delivery & $10 \%$ & $14 \%$ & $29 \%(-49$ to 67$)$ & NS \\
Vacuum extraction & $21 \%$ & $22 \%$ & $5 \%(-58$ to 43$)$ & NS \\
Amniotomy & $47 \%$ & $54 \%$ & $13 \%(-14$ to 34$)$ & NS
\end{tabular}

*Abbreviations defined in glossary; RRR, NNT, and CI calculated from data in article; $\uparrow \mathrm{NS}=$ not significant.

Source of funding: University of Toronto.

For correspondence: $\operatorname{Dr}$ P S McNiven, McMaster University Midwifery Education Programme, St Joseph's Hospital, Fontbonne Building F622,50 Charlton Avvenue East, Hamiltom ON L8N 4A6, Canada. Fax +19055216014.

\section{Commentary}

Past research has shown that women with a mean cervical dilation of $\leqslant 3 \mathrm{~cm}$ on hospital admission have longer lengths of labour, an increased number of intrapartum interventions, and more diagnoses of complicated labour. ${ }^{1}$ To diagnose active labour, some hospitals have established early labour assessment or triage programmes. The study by McNiven et al is the first randomised controlled trial to evaluate such a programme.

Study strengths include the random assignment of women to early labour assessment or direct admission to hospital. The outcomes measured were not only about interventions, labour, and the newborn but also about the women's evaluations of the experience. 2 limitations could have affected study results. Firstly, although there were fewer caesarean section births in the early assessment group, the difference did not reach statis- tical significance. The authors explain that they were not able to calculate a sample size and therefore chose 200 to be a feasible sample size. This sample size may have been too small to detect differences in caesarean delivery rates. Secondly, all caregivers did not comply with the study protocol and therefore a large number of women in the control group were sent home from the labour and delivery unit. Because some women in the control group received the intervention, the 2 groups were more alike than intended.

Assessment area nurses or medical interns conducted vaginal examinations to determine cervical dilation and whether active labour had begun. They also provided support, encouragement, and advice. When it was unclear whether a woman was in labour, the nurse asked the patient to remain in the assessment area for several hours to reassess her labour progress.
When active labour was reached, the nurse transferred the woman to the labour and delivery unit. It is important that nurses assess each woman individually not only to determine onset of labour but also to evaluate their emotional status. Some women, particularly those who are poorly supported, frightened, or who have had past adverse experiences, may benefit from remaining in the hospital setting.

Caroline Homer, RN, CM, MN Research Midwife

Margaret Brock, RN, CM, MN Midwifery Educator

Deborah Matha, RN, CM

Midwifery Manager, Delivery Suite Midwifery Practice and Research Centre St George's Hospital

Sydney, New South Wales, Australia

1 Hemminki E, Simukka R.Eur J Obstet Gynecol Reprod Biol 1986;22:85-94. 